\title{
PROGRESS REPORT OF THE CR-39 NEUTRON PERSONAL MONITORING SERVICE AT PSI
}

\author{
A. Fiechtner*, M. Boschung and C. Wernli \\ Paul Scherrer Institute, CH-5232 Villigen PSI, Switzerland
}

\begin{abstract}
At the Paul Scherrer Institute a personal neutron dosimetry system based on chemically etched CR-39 detectors and automatic track counting is in routine use since the beginning of 1998. The quality of the CR-39 detectors has always been a crucial aspect to maintain a trustable personal neutron dosimetry system. This paper summarises the 7 y experience in routine use. The effect of detector material defects which could lead to false positive neutron doses is described. The potentiality of improving the background statistics by extending the pre-etch time is investigated and involves as a drawback a quite lower sensitivity to thermal neutrons. Furthermore, the impact of small changes in the production process of the detectors on the response to fast and thermal neutrons is shown. For the personal dosimetry at CERN, a new dosimetry concept was launched by combining a CR-39 neutron dosemeter with a Direct-Ion Storage (DIS) dosemeter for photon and beta radiation. The usage period of the CR-39 dosemeters is prolonged now from 3 months up to 12 months. In this context, the long-term behaviour over $1 \mathrm{y}$ of the background track density and the response to Am-Be are described.
\end{abstract}

\section{DETECTOR SYSTEM}

The personal neutron dosimetry system ${ }^{(1)}$ at the Paul Scherrer Institute, which is in routine use since the beginning of 1998 is based on chemically etched CR-39 detectors (PN3) with different radiators (pure polyethylene and polyethylene containing 1\% lithium of natural abundance), and an automatic readout system, the Autoscan $60^{(1)}$. A two-step etching process is used for chemical etching of the detectors. In a first step, the 'pre-etch', the detectors are etched for $30 \mathrm{~min}$ in a mixture of $60 \%$ methanol and $40 \% 6.25 \mathrm{M}$ sodium hydroxide at $70^{\circ} \mathrm{C}$ to polish the surface and to remove alpha particle tracks and scratches. Afterwards, the detectors are etched for $6 \mathrm{~h}$ at $70^{\circ} \mathrm{C}$ in $6.25 \mathrm{M}$ sodium hydroxide. Finally, the detectors are neutralised in a weak hydrochloric acid solution and washed with hot and cold distilled water. The neutron dosemeter at PSI is used for the measurement of thermal and high-energy neutrons (above $\sim 200 \mathrm{keV}$ ).

\section{FALSE NEUTRON DOSES OWING TO MATERIAL DEFECTS}

Since 1998, 30000 CR-39 detectors have been evaluated. For $\sim 1 \%$ of the detectors a neutron dose higher than the detection limit of $0.5 \mathrm{mSv}$ was measured. In most of these cases the measured track density was not due to a neutron irradiation but originates from various material defects. Therefore, the quality of the detector material is still not satisfying. The most serious problem is the nonhomogeneity of the background track density over a whole production sheet ( $\sim 250$ detectors). The sheets

\footnotetext{
*Corresponding author: annette.fiechtner@psi.ch
}

show areas, often they are the same regions from sheet to sheet, where the track density is much higher than the average background track density. Figure 1 shows tracks of an unirradiated detector (typical background tracks) and Figure 2 shows tracks of an neutron-irradiated detector. A material defect can be an easily detectable track cluster (Figure 3) such that a problem arises only when the track cluster extends over a few detectors so that it is not possible to see which detectors are affected. Other material defects makes it more difficult to distinguish between neutron-induced tracks and spurious tracks, because the tracks appear quite normal when evaluated with the Autoscan 60 automatic track counting system. The only hint that the tracks may not be neutron induced is that they appear smaller and less bright than the neutron-induced tracks. Preliminary investigations with an optical microscope show that these tracks are not only on the surface of the detector but throughout the whole thickness. Therefore, these are not etched tracks, but 'bubbles' generated in the production process. This kind of material defect was not only found to occur very often on a certain region of the sheet, but also in the same region; therefore, detectors in this region are now removed beforehand and will not be used for personal dosimetry. Not all described material defects could be eliminated by extending the pre-etch time.

\section{IMPACT OF PRE-ETCH TIME ON BACKGROUND TRACK DENSITY AND SENSITIVITY}

The impact of the pre-etch duration on the background effect and on the sensitivity was investigated. By extending the pre-etch time from 30 to $60 \mathrm{~min}$, the background track density can be decreased by 


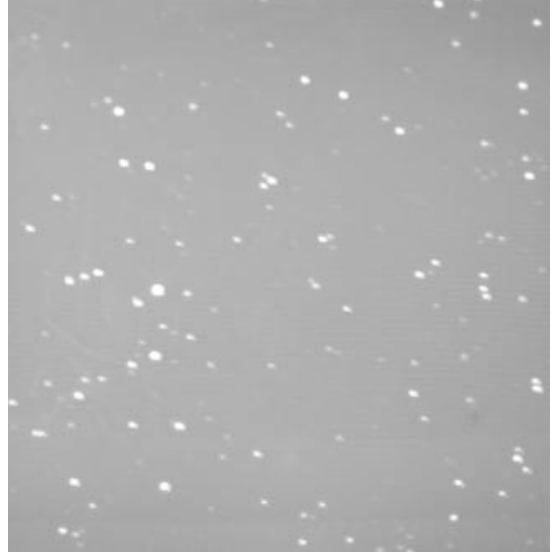

Figure 1. Image of typical background tracks of an unirradiated detector.

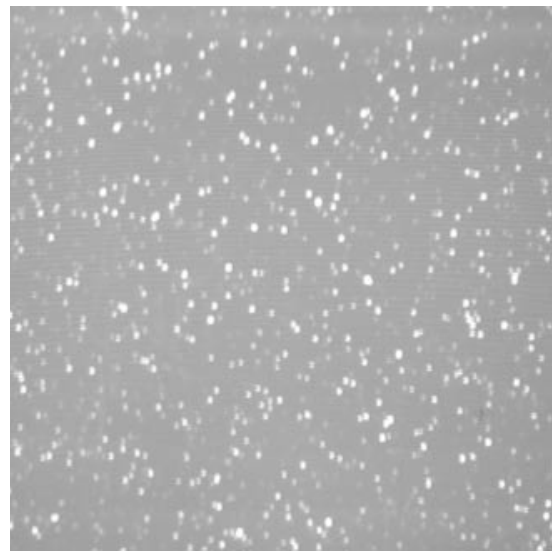

Figure 2. Image of real neutron-induced tracks of an irradiated detector.

factors of 2-4, depending on the age of the detectors and on the storage conditions (which affect radon tracks). The drawback of this procedure is that a measurement of thermal neutrons is not possible any more. For the measurement of thermal neutrons a polyethylene radiator containing $1 \%$ natural lithium is used. In a nuclear reaction of thermal neutrons with ${ }^{6} \mathrm{Li}$, alpha particles and tritons $\left({ }^{3} \mathrm{H}\right)$ are produced. The range of the alpha particles in the detector is too short $(\sim 10 \mu \mathrm{m})$ to be detected, but the tracks of the tritons with a range of $\sim 50 \mu \mathrm{m}$ are not removed by the $30 \mathrm{~min}$ pre-etching. Using a pre-etch time of 45 or $60 \mathrm{~min}$, all tracks owing to tritons are removed, even when a radiator with a high amount of ${ }^{6} \mathrm{Li}$ is used (e.g. TLD-600 chips). The response to fast neutrons is not influenced significantly by a longer pre-etch time. Therefore, when it is not necessary to measure thermal

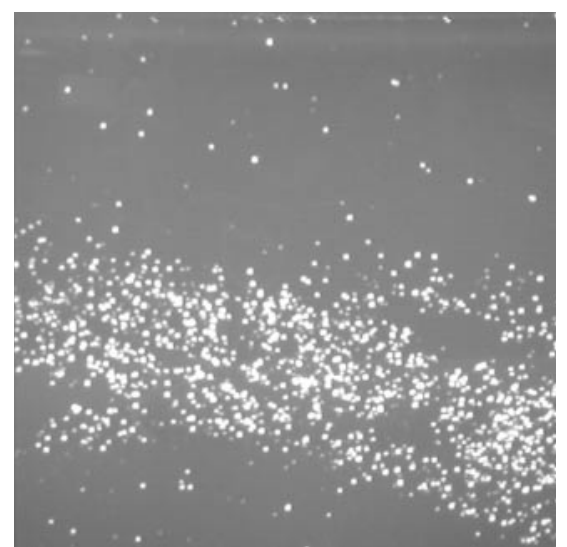

Figure 3. Image of a track cluster of an unirradiated detector.

Table 1. Response of the CR-39 dosemeter to thermal neutrons and thermalised spectra, normalised to Am-Be.

\begin{tabular}{lcc}
\hline $\begin{array}{l}\text { Neutron energy/ } \\
\text { spectrum }\end{array}$ & $\begin{array}{c}\text { Response, } \\
\text { normalised to } \\
\text { Am-Be, pre-2001 } \\
\text { detector material }\end{array}$ & $\begin{array}{c}\text { Response, } \\
\text { normalised to } \\
\text { Am-Be, post-2001 } \\
\text { detector material }\end{array}$ \\
\hline 2.53E-8 MeV & $2.1 \pm 0.2$ & $0.56 \pm 0.20$ \\
SIGMA & $3.0 \pm 0.6$ & $0.45 \pm 0.14$ \\
CANEL & $0.60 \pm 0.10$ & $0.33 \pm 0.03$ \\
\hline
\end{tabular}

Detector material produced before and after the beginning of 2001. Pre-etch time $30 \mathrm{~min}$.

neutrons, a pre-etch time of 60 min appears certainly preferable.

\section{NEUTRON ENERGY DEPENDENCE OF THE RESPONSE}

In 2001, a decrease in the response to thermal neutrons of the detectors was noticed during a research campaign. A consultation with the producer of the detectors (Thermo Electron Corporation) yielded no explanations. Some small changes in the production process were made which were not expected to influence the sensitivity to thermal neutrons (i.e. to tritons). The calibration with thermal neutrons was repeated several times and the lower sensitivity to thermal neutrons for post-2001 detector material was found to be reproducible. The response to thermal neutrons for detector material produced before 2001 was also measured again and agreed with the earlier results.

In Table 1, the responses to thermal neutrons and to some simulated work place field spectra are shown for detector material produced before and after the 


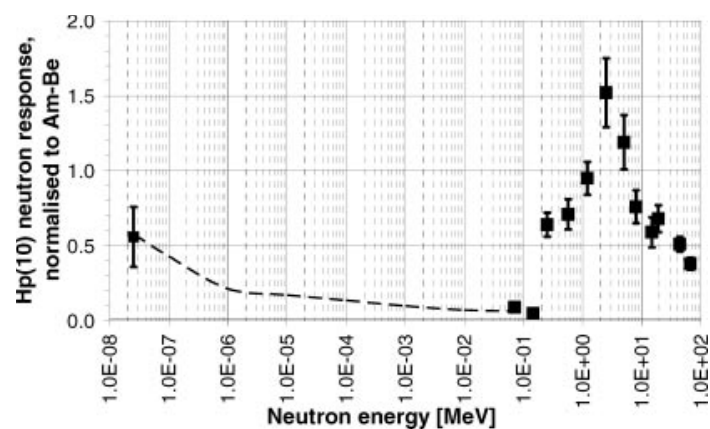

Figure 4. Personal dose equivalent $[H p(10)]$ response as a function of neutron energy, normalised to Am-Be. Detector material produced after the beginning of 2001. Pre-etch time $30 \mathrm{~min}$. The responses to intermediate neutron energies were calculated by means of albedo spectra $^{(5)}$ and cross sections of natural abundance lithium $^{(6)}$. The calculated responses are normalised to the response to thermal neutrons.

beginning of 2001. The irradiations with thermal neutrons were performed at the thermal beam at GKSS $^{(2)}$ in Geesthacht, Germany. Two simulated work place fields were supplied by IRSN (Cadarache, France). These fields presented, respectively, a broad, partly thermalised spectrum, 'Canel' (3), and a highly thermalised spectrum, 'Sigma'(4). The response of post-2001 detectors to thermal neutrons and to simulated work place field spectra decreased by a factor 2-5 compared with pre-2001 detectors, depending on the thermalisation of the spectrum. The energy dependence for the personal neutron dose equivalent for post-2001 detector material is shown in Figure 4. The response to fast neutrons did not change for post-2001 detector material. The identified decrease in thermal neutron response implies that the calibration with thermal neutrons has to be verified more regularly (i.e. at least once a year) for the routine use of the neutron dosimetry system.

\section{LONG-TERM BEHAVIOUR OF BACKGROUND TRACK DENSITY AND SENSITIVITY}

For the personal dosimetry service at CERN, a new dosimetry concept was launched in 2004 by combining a CR-39 neutron dosemeter with a Direct-Ion Storage (DIS) dosemeter for photon and beta radiations. The new dosimetry concept is described in detail elsewhere ${ }^{(7)}$. The combination-dosemeter is kept by the worker for up to $1 \mathrm{y}$. The photon dose is read monthly, and only if the photon dose exceeds $2 \mathrm{mSv}$ per month the dosemeter is replaced immediately and the CR-39 detector is then etched and evaluated. This means that the usage period of the

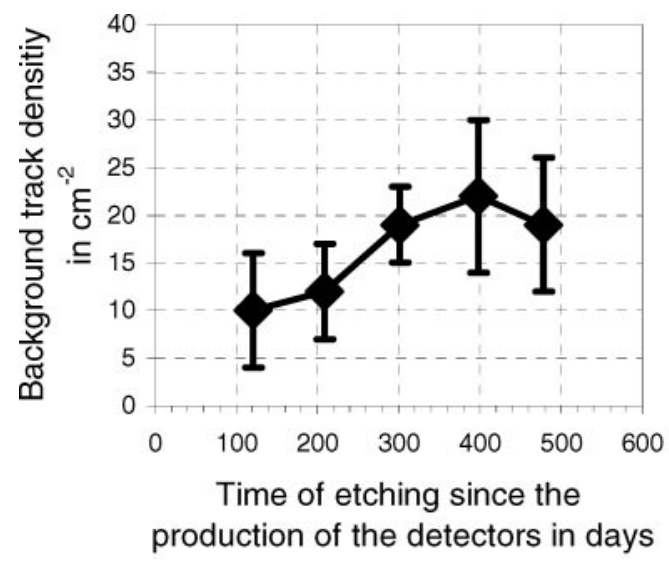

Figure 5. Background track density, as a function of the age of the detectors.

CR-39 dosemeter is extended from 3 months up to 12 months. In this context, the long-term behaviour over $1 \mathrm{y}$ of the background track density and the response to $\mathrm{Am}-\mathrm{Be}$ is described. Since it is not necessary to measure thermal neutrons at CERN, the detectors are processed with a pre-etch time of $60 \mathrm{~min}$. For the determination of the long-term behaviour of the background track density detectors were etched at five different dates during $1 \mathrm{y}$. The sensitivity of an irradiated detector depends on two factors. First, the age of the detector at the time of irradiation and, second, the time between irradiation date and etching date. Therefore, detectors were on the one hand irradiated at the beginning of the 1 -y-experiment and etched at four different dates and on the other hand irradiated at three different dates during $1 \mathrm{y}$.

The detailed test conditions are as follows:

- Production of detectors on Day 0.

- Five dates of etching: Days 121, 209, 301, 398 and 478 after the production of detectors.

- Packing of detectors in dosemeters on Day 127.

- Storage of dosemeters (room temperature, normal pressure, normal atmosphere).

- Three dates of irradiation (Am-Be): Days 129, 296 and 477 after the production of detectors.

- Statistics: 20 background detectors, 6 irradiated detectors for each irradiation date and etching date.

- Pre-etch time $60 \mathrm{~min}$.

The background track density and its standard deviation in relation to the detector age at the time of etching is shown in Figure 5. Over 1 y, the background track density increases by a factor of 2 . The fading of the tracks (before etching) of an irradiated detector in relation to the time between irradiation and etching (Figure 6 ) is 15\% for detectors irradiated 


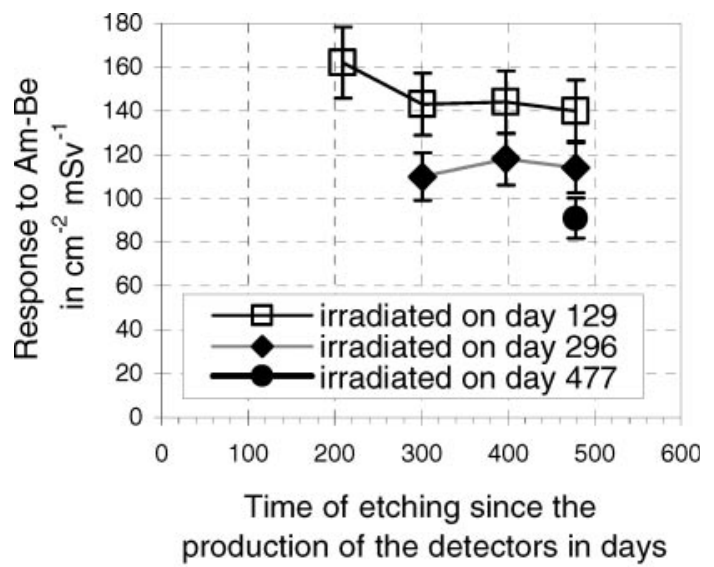

Figure 6. $H \mathrm{p}(10)$ neutron response to $\mathrm{Am}-\mathrm{Be}$, as a function of the age of the detectors and the time of irradiation and etching.

at Day 129 and $0 \%$ for the other irradiated detectors. More pronounced is the decrease of sensitivity in relation to the detector age at the time of irradiation (Figure 6). In this case, the sensitivity decreases in $1 \mathrm{y}$ by a factor of 2 . The decrease is approximately linear. The detection limit for $\mathrm{Am}-\mathrm{Be}$ is calculated by dividing the triple standard deviation of the background track density with the response to Am-Be. Over $1 \mathrm{y}$, the detection limit increases from $\sim 0.1$ up to $\sim 0.3 \mathrm{mSv}$, mainly owing to the decreased response to $\mathrm{Am}-\mathrm{Be}$ when the detector is irradiated at a later date (Figure 7). In routine application, the time of irradiation is normally not known (except for accident circumstances). Therefore, for the routine calculation of the neutron dose a constant irradiation over the whole usage period is assumed. With this assumption, the neutron dose will be overestimated for an irradiation occurring at the beginning of the usage period and underestimated for an irradiation occurring at the end of the period.

\section{CONCLUSIONS}

The personal neutron dosimetry system based on chemically etched CR-39 detectors is in general a stable and sensitive system for monitoring fast neutrons. The crucial point is still the quality of the CR-39 detector material, where surface effects and bulk effects ('bubbles') occur. For the measurement of thermal neutrons it has to be investigated, if the thermal response can be increased by applying a

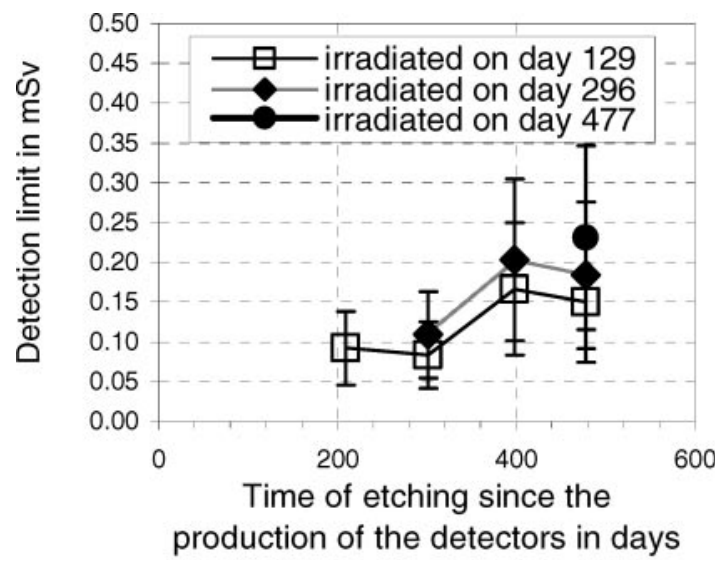

Figure 7. Detection limit for Am-Be irradiation, as a function of the age of the detector and the time of irradiation and etching.

shorter pre-etch time and the use of other converter materials. The results of the long-term experiment are preliminary and further studies on the long time dependence of the sensitivity and fading have to be carried out. The possibilities for the improvement of the decreasing sensitivity need to be investigated.

\section{REFERENCES}

1. Fiechtner, A. and Wernli, C. Individual neutron monitoring with CR-39 at an accelerator centre. Radiat. Prot. Dosim. 85(1-4), 35-38 (1999).

2. Böttger, R., Dietz, E., Friedrich, H., Lagner, F., Luszik-Bhadra, M. and Sosaat, W. Thermal neutron calibration field for radiation protection. GKSS Report 2002/4 (2002)

3. Lacoste, V., Gressier, V. and Bolognese-Milsztajn, T. Monte Carlo simulation of the neutron and dosimetric characteristics at the IRSN Canel/T400 Facility. Radiat. Prot. Dosim. 110(1-4), 123-127 (2004).

4. Muller, H., Gressier, V., Lacoste, V., Lebreton, L. and Pochat, J.-L. Characterization of the thermal neutron field produced by the IRSN Sigma Facility. Radiat. Prot. Dosim. 110(1-4), 136-140 (2004).

5. Alsmiller, R. G., Jr and Barish, J. The calculated response of ${ }^{6} \mathrm{LiF}$ albedo dosimeters to neutrons with energies $\leq 400 \mathrm{MeV}$. Health Phys. 26(1), 13-28 (1974).

6. Neutron cross sections. Vol. 2 (Academic Press, Inc.) (1988).

7. Wernli, C. and Boschung, M. A novel concept and technique for individual monitoring for photon, beta and neutron radiation. In: Proceedings of the IRPA 11, (Madrid, Spain, 23-28 May 2004, paper 3h74) (2004). 